\author{
Caroline A. Sabin a and Jens D. Lundgren ${ }^{\mathrm{b}}$
}

\begin{abstract}
Purpose of review
To review recent published literature around three areas: long-term nonprogression/viral control; predictors of viral load set point/disease progression; and the potential impact of antiretroviral therapy (ART) in early
\end{abstract} HIV infection.

\title{
Recent findings
}

The natural course of untreated HIV infection varies widely with some HIV-positive individuals able to maintain high CD4 cell counts and/or suppressed viral load in the absence of ART. Although similar, the underlying mechanistic processes leading to long-term nonprogression and viral control are likely to differ. Concerted ongoing research efforts will hopefully identify host factors that are causally related to these phenotypes, thus providing opportunities for the development of novel treatment or preventive strategies. Although there is increasing evidence that initiation of ART during primary infection may prevent the immunological deterioration which would otherwise be seen in untreated HIV infection, recent studies do not address the longer term clinical benefits of ART at this very early stage.

\section{Summary}

A better understanding of the relative influences of viral, host, and environmental factors on the natural course of HIV infection has the potential to identify novel targets for intervention to prevent and treat HIV-infected persons.

\section{Keywords}

antiretroviral treatment, disease progression, elite control, long-term nonprogression, natural history, primary HIV infection

\section{INTRODUCTION}

In the early days of the HIV epidemic, knowledge about the natural history of HIV accrued rapidly. However, the widespread use of effective antiretroviral therapy (ART) brought a shift in focus of the research community away from studies of natural history to those of treated infection. Nevertheless, recent years have seen many advances in our knowledge about natural history. For the purposes of this review, we will focus on three areas of relevance to treating clinicians: long-term nonprogression and viral control; predictors of viral load set point and disease progression; and the potential impact of ART in early HIV infection.

\section{LONG-TERM NONPROGRESSORS AND ELITE CONTROLLERS}

The natural course of untreated HIV infection varies widely. The past decade has seen considerable interest in the identification of subgroups of HIV-positive persons who exhibit distinct patterns of disease progression. It is hoped that the information obtained through the identification of such individuals might provide insight for the development of vaccines and novel treatment approaches.

Long-term nonprogressors (LTNP) are individuals who remain asymptomatic for a prolonged period of time off ART with a high CD4 cell count (see reviews by Poropatich and Sullivan and Gaardbo et al. $\left.\left[1,2^{*}\right]\right)$. Although it is widely reported that $1-5 \%$ of the HIV-positive population are LTNP, these estimates are complicated by the fact that there is no standardized definition of a LTNP, and

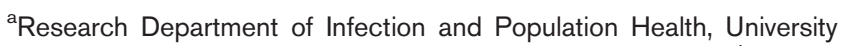
College London (UCL), Royal Free Campus, London, UK and bepartDepartment of Infectious Diseases, Copenhagen University Hospital/ Rigshospitalet and Copenhagen HIV Programme, University of Copenhagen, Copenhagen, Denmark

Correspondence to Professor Caroline A. Sabin, Research Department of Infection and Population Health, UCL, Royal Free Campus, Rowland Hill Street, London NW3 2PF, UK. Tel: +44 2077940500 ext 34752; e-mail: c.sabin@ucl.ac.uk

Curr Opin HIV AIDS 2013, 8:311-317

DOI:10.1097/COH.0b013e328361fa66

This is an open access article distributed under the Creative Commons Attribution License 4.0, which permits unrestricted use, distribution, and reproduction in any medium, provided the original work is properly cited. 


\section{KEY POINTS}

- Despite the existence of several phenotypes associated with slowly progressing HIV infection, it is likely that virtually all HIV-positive individuals will eventually experience disease progression if left untreated.

- Variation in viral characteristics, host defence responses (likely explained by variation in host genetics), and environmental factors may all contribute to the variation in the natural course of HIV infection.

- If concerted research efforts are able to identify host factors that are causally related to viral control, this will provide insight for the development of therapeutic or preventive intervention strategies.

- Further evidence has accrued that initiation of ART during primary infection may prevent the immunological deterioration that is the hallmark of untreated HIV infection, although the longer term clinical benefits of such an approach remain unclear.

- Although there is global consensus of the benefits of initiating ART during chronic infection in those with HIVrelated symptoms and/or a low CD4 cell count, such a consensus has not yet been reached for the initiation of ART in asymptomatic individuals with a CD4 cell count more than 350 cells $/ \mu$ l.

thus definitions used (and the way in which they are applied, particularly in the presence of varying follow-up and irregularly measured CD4 cell counts) differ widely (Table 1) $\left[3-5,6^{\boldsymbol{\prime}}, 7,8\right]$. For example, Madec et al. [3] identified asymptomatic individuals who remained off ART for more than 8 years with a CD4 cell count more than 500 cells/ $\mu$ l; using this definition, $9 \%$ of their clinic population were identified as LTNP. Using a similar definition but with only 7 years of follow-up, Okulicz et al. [4] reported a prevalence of $5.02 \%$ in a military cohort. In contrast, only $0.4 \%$ of patients in the French Hospital's Database on HIV were identified as LTNP [5]. In a UK study, Mandalia et al. [6"'] identified ART-naive asymptomatic individuals infected with HIV for more than 7 years. Of 312 such patients, only 50 had stable CD4 cell counts, with only 13 having CD4 cell counts consistently in the normal range. Thus, LTNP represented only $0.2 \%$ of patients attending for care, a far lower rate than that reported by Okulicz et al., presumably because of the additional requirement that individuals had stable CD4 cell counts.

LTNP status can be lost, and thus the reported prevalence of LTNP within a study will depend on the required period of follow-up. In Madec's study [3], LTNP status was lost after 8 years in 36 of the 60 LTNP; loss of LTNP status was generally because of declining CD4 cell counts and initiation of ART, although a small number of individuals experienced Centers for Disease Control stage B/C disease. Predictors of loss of LTNP status were a high baseline HIV DNA level and a more rapid increase in HIV DNA over the first years of follow-up, suggesting the presence of ongoing (but low-grade) viral replication. Indeed, HIV RNA levels in plasma increased by $0.04 \log _{10}$ copies $/ \mathrm{ml}$ per year over the first 8 years after diagnosis. When the required period of followup was increased from 7 to 10 years in a military cohort [4], the prevalence of LTNP status dropped from 5 to $2 \%$. The fact that an individual's LTNP status can change has led some to suggest that rather than representing a distinct group of HIV-positive individuals, LTNP are more likely to represent individuals at one tail end of a Normal distribution [6"']. As such, it is likely that virtually all HIVpositive persons will eventually experience disease progression if left untreated.

More recently, interest has shifted towards the identification of individuals who are able to suppress HIV replication to such an extent that viral load levels remain undetectable in the absence of ART [9]. These individuals are generally referred to as elite controllers or viral controllers. In the military cohort described by Okulicz et al. [4] elite controllers were defined as ART-naive patients infected with HIV for more than 12 months with at least three longitudinal undetectable HIV RNA determinations. Individuals were allowed to have occasional HIV RNA levels up to 1000 copies/ml as long as these episodes represented the minority of all determinations. These elite controllers were distinguished from viremic controllers in whom the majority of viral loads were in the range $1000-2000$ copies $/ \mathrm{ml}$. In total, $0.6 \%$ of 4586 individuals were identified as elite controllers and $3.3 \%$ as viremic controllers. Virological control was established a median of 1 year after seroconversion, lasted for 846 and 1085 days in elite controllers and viremic controllers, respectively, and was associated with a reduced risk of clinical progression. Interestingly, although elite controllers experienced an initial CD4 cell count increase followed by stabilization, viremic controllers generally experienced a loss of CD4 cells. Goujard et al. [10] confirmed that elite controllers status is established early after primary infection in the Agence Nationale de Recherche sur le Sida PRIMO cohort.

Although there is clearly overlap between the LTNP and elite controller groups, not all LTNP have a suppressed viral load, and not all elite controllers have high CD4 cell counts. Furthermore, LTNP status is not necessarily protective against clinical progression. An early study from the 


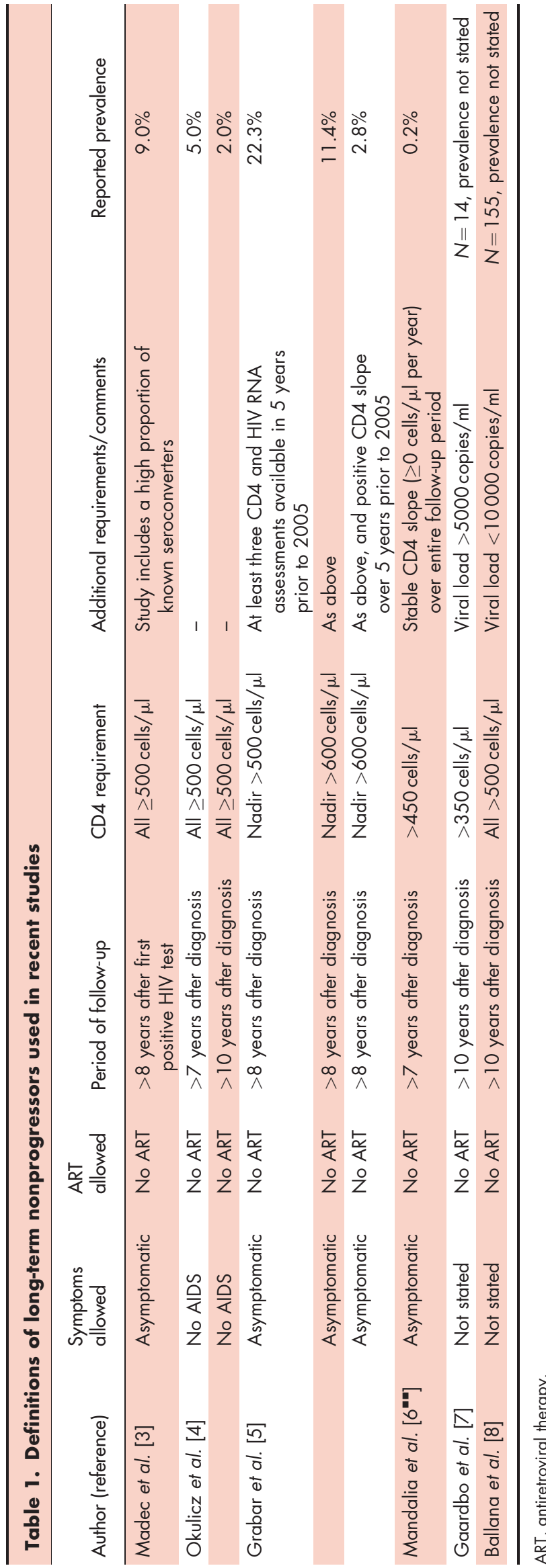

CASCADE group [11] suggested that 15 and $7 \%$ of elite controllers infected with HIV for more than 16 years had CD4 cell counts less than 350 cells/ $\mu$ l or AIDS, respectively. A more recent study from the group [12] demonstrated that the proportion of elite controllers with at least one CD4 cell count less than 500 cells/ $\mu \mathrm{l}$ ranged from 45 to $53 \%$, depending on the definition of elite controllers. Sedaghat et al. [13] noted that CD4 slopes in elite controllers varied substantially with rates of CD4 loss of up to 53 cells $/ \mu$ l per year in some individuals. Using a highly sensitive viral load assay, Pereyra et al. [14] reported that the median viral load was 2 copies $/ \mathrm{ml}$ in 90 elite controllers. Low-level viremia was present in the majority of elite controllers; CD4 loss was more common among those with low-level viremia than in those without detectable virus. Boufassa et al. [15] reported that clinical and immunological progression in elite controllers was restricted to those experiencing viral load 'blips'.

More recently, Groves et al. [16"'] identified ART-naive patients who had maintained a viral load less than 2000 copies/ml for more than 12 months. Typical controllers had an average recent CD4 cell count more than 450 cells/ $\mu$ l (2.1\% of population), whereas discord controllers had an average recent CD4 cell count less than 450 cells/ $\mu l(0.6 \%)$. Thus, in this study, the term discord controller was used to identify individuals who had experienced a loss of CD4 cells despite viral control. There were no significant differences in viral load or demographic factors between the two groups. Interestingly, there was a suggestion of a higher frequency of infection with subtype $C$ virus in discord controllers $(40 \%$ of patients) compared with the entire clinic population of whom $25.1 \%$ were infected with subtype C; whether this overrepresentation relates to specific features of subtype $C$ virus itself, or whether subtype $\mathrm{C}$ is merely a marker of infection in certain regions of the world with specific host genetic and environmental factors is, however, unclear.

As with LTNP, several studies have attempted to identify factors associated with elite controller status. Yang et al. [17] considered the relative and absolute numbers of naive $\mathrm{T}$-cells in a cohort of elite controllers with normal or declining CD4 cell counts and in ART-treated individuals. The relative proportions of naive CD4 and CD8 T cells were reduced in elite controllers, resembling the patterns seen in individuals with untreated progressive HIV infection. The authors concluded that loss of naive CD4 T cells is a universal feature of elite controllers, despite the ability of such individuals to maintain undetectable viral loads. Chen et al. [18] suggested, based on in-vitro experimentation, that CD4 naive lymphocytes from elite controllers were less 
susceptible to HIV infection than such lymphocytes from progressors or uninfected individuals. This specific feature was linked with upregulation of a cellular kinase (p21). Mahnke et al. [19"] compared patients maintaining low levels of viremia (controllers) with those experiencing disease progression within 2 years of diagnosis (fast progressors) and with progressive disease not requiring ART (slow progressors). Although beneficial human leukocyte antigen (HLA) types (HLA-B $* 27, \mathrm{~B} * 57$, and $\mathrm{B} * 58$ ) were seen more commonly in controllers (57\%) they were also expressed by $23 \%$ of slow progressors. Progressors were more likely to be coinfected with GB virus $C$ than controllers, coinfection with which has previously been reported by some to be associated with a slower rate of disease progression in HIV infection [20], but the CCR5 $\Delta 32$ mutation was similarly distributed across the groups. Plasma HIV viral load did not differ between progressors, but cellassociated viral load was elevated in fast progressors and lowered in controllers. Although the frequency of $\mathrm{CD} 8^{+} \mathrm{CD}^{+} \mathrm{T}$ cells was a strong predictor of disease progression in the first year after HIV infection, and was sufficient to distinguish progressors from controllers, this measurement alone could not differentiate between fast and slow progressors.

As the two groups of individuals appear to be clinically distinct, suggesting differences in the processes that lead to long-term nonprogression and elite control, several research groups have attempted to investigate whether there are any demographic or biological differences between these two patient groups [4]. Groves et al. [16"'] reported a more marked depletion of the naive T-cell subset in discord controllers than in typical controllers but a trend towards increased activated effector memory CD4 cells in typical controllers. CD8 T-cell activation was increased to a similar level (compared with noncontrollers) in both controller groups. The authors concluded that despite the lower CD4 cell counts in discord controllers, their CD8 T-cell activation pattern more closely resembled that of typical controllers. As with other studies, the discord controllers had higher viral DNA loads than the typical controllers, suggesting continued viral replication in this subgroup.

Shaw et al. [21] compared viral controllers (individuals with viral load $<1000$ copies/ml for $>5$ years) to viremic slow progressors (individuals with a viral load $>10000$ copies/ml but who had maintained a CD4 cell count $>500$ cells/ $\mu$ l for $>7$ years) and viremic progressors (individuals infected for a similar time with viral load $>10000$ copies/ml but CD4 cell count $<500$ cells $/ \mu \mathrm{l})$. Viremic slow progressors had higher levels of markers of mucosal immune activation and low numbers of mucosal Tregs, suggesting that factors other than immune activation account for this phenotype. Gaardbo et al. [7] reported that LTNP had a higher frequency of activated CD4 and CD8 cells compared with viral controllers, but similar levels to progressors. Ballana et al. [8] confirmed results from other studies [22] showing that a single nucleotide polymorphism $35 \mathrm{~kb}$ upstream of the $H L A-C$ gene $(-35 \mathrm{C} / \mathrm{T})$ is associated with LTNP status.

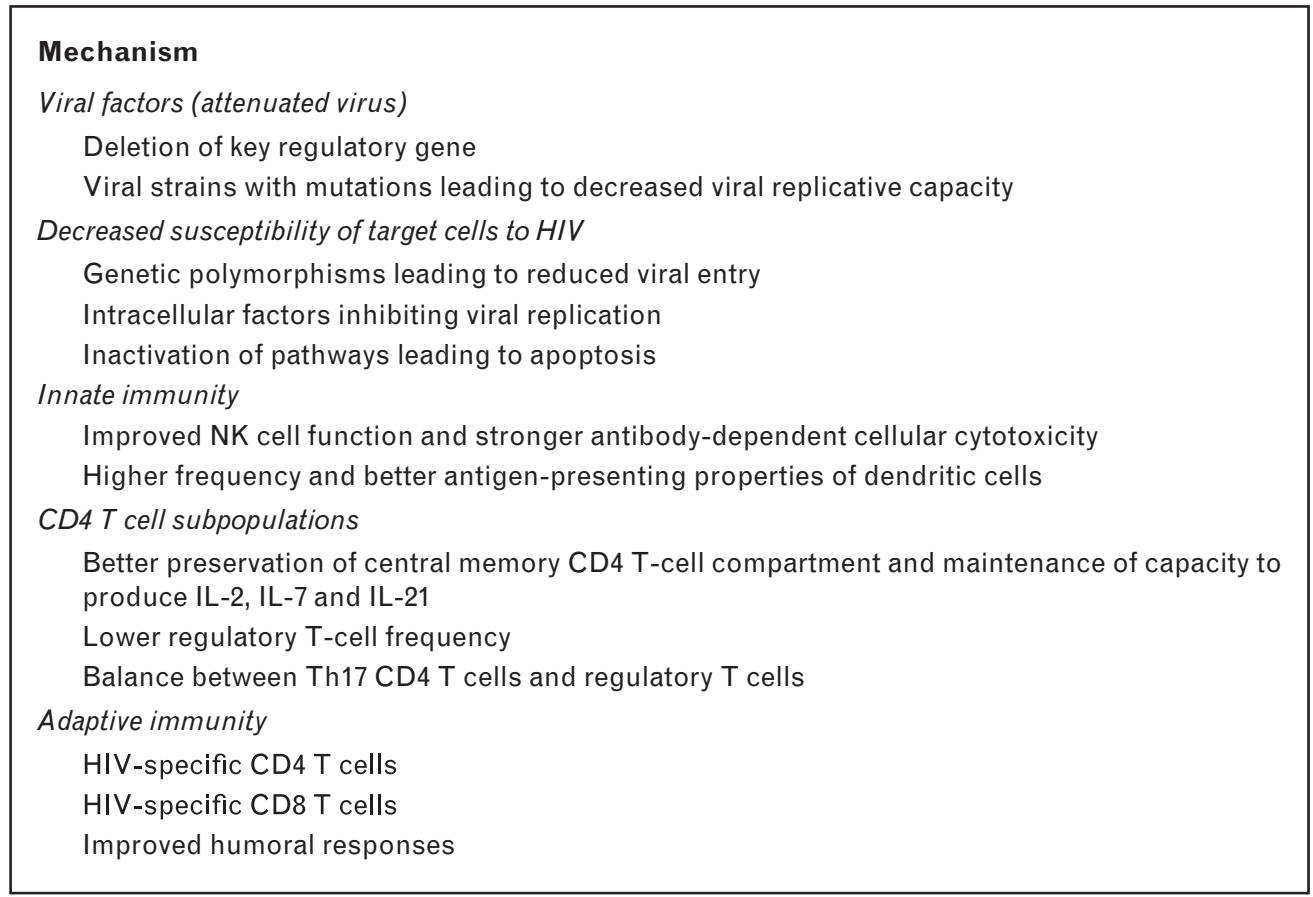

FIGURE 1. Potential mechanisms of viral suppression in HIV controllers. Adapted from [24]. 
HIV-specific CD4 activation is a hallmark of viral control [23] but, as outlined above and reviewed recently [24] (see Fig. 1), many other host factors have been linked with this phenotype, including cellular restriction factors such as APOBEC, tetherin, and the recently identified SAMHD1 $[25,26]$. In addition, several viral factors may also play a role, including deletions or mutations with the viral genes [27] that may have an impact on the ability of the virus to replicate. Concerted ongoing research efforts will hopefully clarify whether any of these host factors are causally related to viral control or merely reflect intrinsic variations in the ability of the virus to replicate. If any host or viral factors do have an important influence on viral replication, such a discovery will open a field of possibilities aimed at enhancing or mimicking these host factors as part of a therapeutic or preventive intervention strategy.

\section{OTHER PREDICTORS OF VIRAL LOAD SET POINT AND CD4 LOSS}

The possibility that there may be a link between the viral load set point and the viral load of the infecting partner was raised by Hecht et al. [28] who demonstrated that in 24 transmission pairs, the viral load in the donor was closely associated with the viral load at presentation in the seroconverting partner (correlation coefficient $=0.55$ ). Using a novel phylogenetic approach to determine heritability, Alizon et al. [29] concluded that up to half the variance in the viral load set point among individuals in the Swiss HIV Cohort Study could be heritable from their infecting partners. These observations support the notion that HIV has varying intrinsic replicative capacity and suggest that this feature is maintained after transmission.

Predictors of the viral load set point were investigated by Lingappa et al. [30"'] in 141 African seroconverters. In multivariable analysis, higher viral loads in the source partners were associated with higher viral load set points in the seroconverters. The proportion of variation in set point that could be attributed to the viral load of the source partner, after controlling for other factors, was $6 \%$. Despite this low proportion, the authors concluded that the source partner viral load was the most significant predictor of the viral load set point in the seroconverter. Yue et al. [31"'] also noted the relatively small proportion of variance in the viral load set point that could be explained by the viral load in the source partner. In an analysis of 195 transmission pairs from Zambia, the viral load in source partners explained only around $2 \%$ of the variance in viral load set points of seroconverters.
Overall, the viral load set point was a function of the source partner viral load, the sex of the seroconverter, the HLA class I alleles of the seroconverter, and the sharing of HLA-I alleles between partners in a transmission pair. Together, these factors accounted for up to $37 \%$ of variance in the viral load set point. Roberts et al. [32] reported that the concentration of five plasma cytokines (IL-12p40, IL-12p70, IFN- $\gamma$, IL-7, and IL-15) predicted $66 \%$ of the variation in viral load set point in 40 South African women. Grinsztejn et al. [33"] reported that women in the Prospective Evaluation of Antiretrovirals in Resource-Limited Settings (PEARLS) study had a lower mean preART viral load than men; whereas the sex difference was related to the CD4 cell count, it was independent of country and persisted in those with a CD4 cell count less than 200 cells/ $\mu$ l.

Other predictors of disease progression include transmission of resistant strains of HIV [34] and the envelope diversity of the virus in the individual after seroconversion [35"]. In the latter study, viral diversity at 1-year postseroconversion was associated with accelerated progression to clinical AIDS or a low CD4 cell count, although not with the viral load set point itself. The authors could not determine whether viral diversity is a direct cause of immunodeficiency, or a consequence of the individual's response to infection. In a small study of 50, chronically infected, asymptomatic, ARTnaive adults from the United Kingdom and China [36], the antiviral inhibitory capacity of $\mathrm{CD}^{+} \mathrm{T}$ cells was highly predictive of CD4 cell loss in early HIV infection. Audige et al. [37] reported that fast progressors (those with a CD 4 cell count $<200$ cells/ $\mu 1$ within 7.5 years) had significantly lower postseroconversion CD4 cell counts than either intermediate ( $7.5-12$ years) or slow ( $>12$ years) progressors; fast progressors had cell-surface CD4 densities that decreased more rapidly than slow progressors.

\section{ANTIRETROVIRAL THERAPY DURING PRIMARY HIV INFECTION}

There is global consensus that there is a favourable benefit: risk ratio for initiating ART in those with HIV-related symptoms or with a CD4 cell count less than 350 cells $/ \mu$ l. Because of the risk of disease progression in these individuals, the benefits of ART outweigh any potential risks of adverse drug reactions. Such a favourable benefit: risk ratio has not yet been established for initiating ART earlier in the course of infection in asymptomatic individuals.

Several recent publications provide further evidence that initiation of ART during primary infection may prevent the immunological deterioration, 
which would otherwise be seen in untreated HIV infection. In one observational study, $64 \%$ of individuals who initiated ART during primary infection maintained a CD4 cell count more than 900 cells/ $\mu \mathrm{l}$ compared with only $34 \%$ of those who deferred ART to a later time [38"]. In the Short Pulse Anti Retroviral Therapy at HIV Seroconversion (SPARTAC) trial, 366 adults with primary infection were randomized to receive either short-term ( 12 weeks) or longer term (48 weeks) immediate ART, or to defer ART until the CD4 cell count dropped to less than 350 cells/ $\mu$ l [39"']. Immediate use of ART reduced the chance of experiencing a CD4 cell count less than 350 cells/ $\mu \mathrm{l}$ while the patient remained on ART, but not beyond the duration of treatment. Using data from the observational CASCADE collaboration, Zugna et al. [40"] reported that although individuals initiating treatment within 12 months of seroconversion were more likely to interrupt therapy than those initiating treatment during chronic infection, rates of virological failure and treatment change were similar between the two groups.

Although these studies demonstrate that ART can prevent the deterioration of the immune system which would otherwise be seen without treatment, they do not address whether those initiating ART during primary infection experience any long-term clinical benefit (in terms of reduced morbidity or mortality) from this treatment, and thus whether allowing CD4 cell counts to fall to lower levels will result in any appreciable negative consequences over either the shortterm or longterm. Unfortunately, such information can only be obtained from clinical endpoint studies with the requirement for substantially larger sample sizes. The ongoing Strategic Timing of Anti-Retroviral Treatment (START) study [41] aims to address this question.

\section{CONCLUSION}

Although the clinical, immunological, and virological course of untreated HIV infection is variable, few persons followed for more than 8-10 years remain without any evidence of disease progression. Variation in viral characteristics, host defence responses (likely explained by variation in host genetics), and environmental factors may all contribute to the variation in the natural course of HIV infection. A better understanding of the relative influence of these factors is emerging. This line of research has the potential to identify novel targets for intervention to prevent and treat HIV-infected persons.

\section{Acknowledgements}

None.

\section{Conflicts of interest}

JDL is a member of the Executive and Scientific Steering Committees for the INSIGHT Network which is currently conducting the START trial. CAS has provided statistical input to various study designs from the INSIGHT group. There are no relevant financial conflicts of interest.

\section{REFERENCES AND RECOMMENDED READINC}

Papers of particular interest, published within the annual period of review, have been highlighted as:

- of special interest

- - of outstanding interest

Additional references related to this topic can also be found in the Current

World Literature section in this issue (pp. 353-354).

1. Poropatich K, Sullivan DJ. Human immunodeficiency virus type 1 long-term nonprogressors: the viral, genetic and immunological basis for disease nonprogression. J Gen Virol 2011; 92:247-268.

2. Gaardbo JC, Hartling HJ, Gerstoft J, Nielsen SD. Thirty years with

- HIV infection-nonprogression is still puzzling: lessons to be learned from controllers and long-term nonprogressors. AIDS Res Treatment 2012. doi: $10.1155 / 2012 / 161584$.

A very detailed review of the long-term nonprogressor and viral controller phenotypes.

3. Madec Y, Boufassa F, Avettand-Fenoel V, et al. Early control of HIV-1 infection in long-term nonprogressors followed since diagnosis in the ANRS SEROCO/HEMOCO cohort. J Aquir Imm Defic Syndr 2009; 50:19-26.

4. Okulicz JF, Marconi VC, Landrum ML, et al. Clinical outcomes of elite controllers, viremic controllers, and long-term nonprogressors in the US Department of Defense HIV Natural History Study. J Infect Dis 2009; 200:1714-1723.

5. Grabar S, Selinger-Leneman $\mathrm{H}$, Abgrall $\mathrm{S}$, et al. Prevalence and comparative characteristics of long-term nonprogressors and HIV controller patients in the French Hospital Database on HIV. AIDS 2009; 23:1163-1169.

6. Mandalia S, Westrop SJ, Beck EJ, et al. Are long-term nonprogressors very

- slow progressors? Insights from the Chelsea and Westminster HIV cohort, 1988-2010. PLoS One 2012; 7:e29844.

This study provides a very detailed description of some of the difficulties encountered when attempting to quantify the number HIV-positive individuals that meet criteria for long-term nonprogression.

7. Gaardbo J, Ronit A, Hartling $\mathrm{H}$, et al. HIV-infected viremic long-term nonprogressors and controllers display different immunological mechanisms for preserved CD4+ cell counts. Abstract P166, Eleventh International Congress on Drug Therapy in HIV infection, Glasgow, 11-15 November, 2012.

8. Ballana $E$, Ruiz-de Andres A, Mothe B, et al. Differential prevalence of the HAL-C -35 CC genotype among viremia long term nonprogressor and elite controller HIV + individuals. Immunobiol 2012; 217:889-894.

9. Okulicz JF, Lambotte O. Epidemiology and clinical characteristics of elite controllers. Curr Opin HIV AIDS 2011; 6:163-168.

10. Goujard C, Chaix M-L, Lambotte $O$, et al. Spontaneous control of viral replication during primary HIV infection: when is 'HIV Controller' status established? Clin Infect Dis 2009; 49:982-986.

11. Madec $Y$, Boufassa F, Porter K, Meyer L. Spontaneous control of viral load and CD4 cell count progression among HIV-1 seroconverters. AIDS 2005; 19:2001-2007.

12. Olson A, Meyer L, Prins M, et al. Proportion of HIV elite controllers and loss of elite status according to two commonly used definitions. Poster 290. In: Presented at $19^{\text {th }}$ Conference on Retroviruses and Opportunistic Infections; 5-8 March 2012, Seattle USA; 2012.

13. Sedaghat $A R$, Rastegar $D A, O^{\prime} C o n n e l l ~ K A$, et al. $T$ cell dynamics and the response to HAART in a cohort of HIV-1-infected elite suppressors. Clin Infect Dis 2009; 49:1763-1766.

14. Pereyra F, Palmer S, Miura T, et al. Persistent low-level viremia in HIV-1 elite controllers and relationship to immunologic parameters. J Infect Dis 2009; 200:984-990.

15. Boufassa F, Saez-Cirion A, Lechenadec J, et al. CD4 dynamics over a 15 yearperiod among HIV controllers enrolled in the ANRS French Observatory. PLoS One 2011; 6:e18726.

16. Groves KC, Bibby DF, Clark DA, et al. Disease progression in HIV-1-infected - viremic controllers. J Acquir Imm Defic Syndr 2012; 61:407-416.

An interesting study that aims to determine whether there are any characteristics that differ between viral controllers who do and do not experience CD4 cell count loss and between these controllers and those who fail to suppress viraemia.

17. Yang $\mathrm{Y}, \mathrm{Al}-\mathrm{Mozaini} \mathrm{M}$, Buzon MJ, et al. CD4 T-cell regeneration in HIV-1 elite controllers. AIDS 2012; 26:701-706. 
18. Chen $\mathrm{H}$, Li C, Huang J, et al. CD4+ T cells from elite controllers resist HIV-1 infection by selective upregulation of p24. J Clin Invest 2011; 121:15491560.

19. Mahnke YD, Song $\mathrm{K}$, Sauer MM, et al. Early immunologic and

- virologic predictors of clinical HIV-1 disease progression. AIDS. (in press).

A small, but detailed study to identify whether any host factors can differentiate

between viral controllers and those that exhibit disease progression.

20. Tillmann $H L$, Heiken $H$, Knapik-Botor $A$, et al. Infection with $G B$ virus $C$ and reduced mortality among HIV-infected patients. New Engl J Med 2001; 345:715-724.

21. Shaw JM, Hunt PW, Critchfield JW, et al. HIV+ viremic slow progressors maintain low regulatory $T$ cell numbers in rectal mucosa but exhibit high T cell activation. AIDS Res Hum Retrovir 2013; 29:172177.

22. Garcia-Merino I, de Las Cuevas N, Himenez JL, et al. The Spanish HIV BioBank: a model of cooperative HIV research. Retrovirol 2009; 6:27.

23. Hunt PW, Hatano $H$, Sinclair $E$, et al. HIV-specific CD4+ T-cells may contribute to viral persistence in HIV controllers. Clin Infect Dis 2011; 52:681-687.

24. Theze J, Chakrabarti LA, Vingert B, et al. HIV controllers: a multifactorial phenotype of spontaneous viral suppression. Clin Immunol 2011; 141: $15-30$.

25. Want $Y$, Whittall $T$, Rahman $D$, et al. The role of innate APOBEC3G and adaptive AID immune responses in HLA-HIV/SIV immunised SHIV infected macaques. PLoS One 2012; 7:e34433.

26. Ayinde D, Casartelli N, Schwartz O. Restricting HIV the SAMHD1 way: through nucleotide starvation. Nat Rev 2012; 10:675-680.

27. Cruz NVG, Amorim R, Oliveira FE, et al. Mutations in the nef and vif genes associated with progression to AIDS in elite controller and slow-progressor patients. J Med Virol 2013; 85:563-573.

28. Hecht FM, Hartogensis W, Bragg L, et al. HIV RNA level in early infection is predicted by viral load in the transmission source. AIDS 2010; $24: 941-$ 945.

29. Alizon S, von Wyl V, Stadler T, et al. Phylogenetic approach reveals that virus genotype largely determines HIV set-point viral load. PLoS Pathog 2010; 6:e1001123.

30. Lingappa JR, Thomas KK, Hughes JP, et al. Partner characteristics predicting

- HIV-1 set point in sexually acquired HIV-1 among African seroconverters. AIDS Res Hum Retrovir 2013; 29:164-171.

The study authors identify that the source partner plasma viral load level is the most significant predictor of the viral load set point in 141 African seroconverters. They also identified several other predictors of the viral load set point, including acyclovir use, bacterial vaginosis in female source partners, and circumcision in male source partners.
31. Yue L, Prentice HA, Farmer $P$, et al. Cumulative impact of host and viral factors .1. on HIV-1 viral-load control during early infection. J Virol 2013; 87:708-715. The authors demonstrate that together, the viral load in the source partner, the sex of the newly-infected partner, specific HLA class I alleles in the newly-infected partner, and sharing of HLA-I alleles between partners in a transmission pair could account for up to $37 \%$ of the variance observed in the newly-infected individual's viral load set point.

32. Roberts L, Passmore J-AS, Williamson C, et al. Plasma cytokine levels during acute HIV-1 infection predict HIV disease progression. AIDS 2010; 24:819-831.

33. Grinsztejn B, Smeaton $L$, Barnett $R$, et al. Sex-associated differences in

- preantiretroviral therapy plasma HIV-1 RNA in diverse areas of the world vary by CD4 ${ }^{+}$T-cell count. Antivir Ther 2011; 16:1057-1062.

Interesting data to suggest that women in resource-limited settings have lower viral load set points than men.

34. Harrison L, Castro $H$, Cane $P$, et al. The effect of transmitted HIV-1 drug resistance on pretherapy viral load. AIDS 2010; 24:1917-1922.

35. Rachinger A, Kootstra NA, Gijsbers EF, et al. HIV-1 envelope diversity 1 year

- after seroconversion predicts subsequent disease progression. AIDS 2012; 26:1517-1522

The author provides interesting data to suggest that viral diversity soon after HIV infection is associated with progression to AIDS and AIDS-related death.

36. Yang $\mathrm{H}, \mathrm{Wu} \mathrm{H}, \mathrm{Hancock} \mathrm{G}$, et al. Antiviral inhibitory capacity of $\mathrm{CD} 8+\mathrm{T}$ cells predicts the rate of CD4+ T-cell decline in HIV-1 infection. J Infect Dis 2012; 206:552-561.

37. Audige $A$, Taffe $P$, Rickenbach $M$, et al. Low postseroconversion CD4 count and rapid decrease of CD4 density identify $\mathrm{HIV}^{+}$fast progressors. AIDS Res Hum Retrovir 2010; 26:997-1005.

38. Le T, Wright EJ, Smith DM, et al. Enhanced CD4+ T-cell recovery with earlier HIV-1 antiretroviral therapy. N Engl J Med 2013; 368:218-230.

An observational analysis that provides supportive data on the benefits of treatment during early HIV infection.

39. SPARTAC Trial Investigators. Short-course antiretroviral therapy in primary

n. HIV infection. N Engl J Med 2013; 368:207-217.

The first randomised controlled trial to demonstrate the value of AVT during primary

HIV infection for the prevention of immunological decline.

40. Zugna D, Geskus RB, De Stavola B, et al. Time to virological failure, treatment

- change and interruption for individuals treated within 12 months of HIV seroconversion and in chronic infection. Antivir Ther 2012; 17:1039-1048.

A detailed analysis from the CASCADE Collaboration of seroconverter cohorts that suggests similar rates of virological failure in individuals initiating treatment soon after seroconversion to those initiating during chronic infection.

41. Babiker AG, Emery S, Fatkenheuer $G$, et al. Considerations in the rationale, design and methods of the Strategic Timing of AntiRetroviral Treatment (START) study. Clin Trials (in press). 